\title{
Nonlinear potentials, local solutions to elliptic equations and rearrangements
}

\author{
ANDREA CIANCHI
}

\begin{abstract}
A sharp rearrangement estimate for the nonlinear Havin-Maz'ya potentials is established. In particular, this estimate leads to a characterization of those rearrangement invariant spaces between which the nonlinear potentials are bounded. In combination with results from [24] and [18], it also enables us to derive local bounds for solutions to quasilinear elliptic PDE's and for their gradient in rearrangement form. As a consequence, the local regularity of solutions to elliptic equations and for their gradient in arbitrary rearrangement invariant spaces is reduced to one-dimensional Hardy-type inequalities. Applications to the special cases of Lorentz and Orlicz spaces are presented.
\end{abstract}

Mathematics Subject Classification (2010): 31C15 (primary); 35B45 (secondary).

\section{Introduction}

The Riesz potential of order $\alpha \in(0, n)$ of a locally integrable function $f$ in $\mathbb{R}^{n}$, $n \geq 1$, is defined as

$$
\mathbf{I}_{\alpha} f(x)=\int_{\mathbb{R}^{n}} \frac{f(y)}{|x-y|^{n-\alpha}} d y \quad \text { for } x \in \mathbb{R}^{n} .
$$

The operator $I_{\alpha}$ plays a role in various branches of analysis, including potential theory, harmonic analysis, Sobolev spaces, partial differential equations. The link to the theory of elliptic PDE's is classical. Indeed, if $n>2$ and $\omega_{n}$ denotes the measure of the unit ball in $\mathbb{R}^{n}$, then the function $\frac{1}{n(n-2) \omega_{n}} \mathbf{I}_{2} f(x)$ is the Newtonian potential in $\mathbb{R}^{n}$, namely the unique solution decaying to 0 at infinity to the Poisson equation

$$
-\Delta u=f \quad \text { in } \mathbb{R}^{n} .
$$

As a consequence, norm estimates for the solution $u$ to (1.2), and for its derivatives, in terms of $f$ are reduced to boundedness properties of the operator $\mathbf{I}_{\alpha}$ for suitable $\alpha$. The study of these properties is a primary issue in harmonic analysis. 
In particular, thanks to a convolution inequality of O'Neil [33], the boundedness of $\mathbf{I}_{\alpha}$ between any function spaces whose norm depends only on integrability properties of functions - the so-called rearrangement invariant spaces - is reduced to one-dimensional Hardy-type inequalities in the spaces in question. O'Neil's result entails that a positive constant $C=C(\alpha, n)$ exists such that

$$
\left(\mathbf{I}_{\alpha} f\right)^{*}(s) \leq C \int_{s}^{\infty} r^{-1+\frac{\alpha}{n}} f^{* *}(r) d r \quad \text { for } s>0,
$$

for every measurable function $f: \mathbb{R}^{n} \rightarrow \mathbb{R}$ fulfilling

$$
|\{x:|f(x)|>t\}|<\infty \quad \text { for } t>0,
$$

and making the right-hand side of (1.3) finite. Here, $|G|$ stands for the Lebesgue measure of a set $G \subset \mathbb{R}^{n}, f^{*}:[0, \infty) \rightarrow[0, \infty]$ denotes the decreasing rearrangement of $f$, defined as

$$
f^{*}(s)=\sup \left\{t \geq 0:\left|\left\{x \in \mathbb{R}^{n}:|f(x)|>t\right\}\right|>s\right\} \quad \text { for } s \geq 0,
$$

and $f^{* *}(s)=\frac{1}{s} \int_{0}^{s} f^{*}(r) d r$ for $s>0$. In other words, $f^{*}$ is the (unique) non increasing, right-continuous function in $[0, \infty)$ equimeasurable with $f$, and $f^{* *}$ is a maximal function of $f^{*}$.

Bounds in terms of Riesz potentials for solutions to more general second and higher-order linear elliptic equations are also available. Even more interestingly, contributions in recent years $[17,18,24,25,32,35,36,40]$ have pointed out that, in analogy with the linear theory, the study of pointwise properties of solutions to nonlinear elliptic (and parabolic) partial differential equations in customary classes is related to that of suitable nonlinear potential operators. A prototype of the relevant differential equations is the $p$-Laplace equation

$$
-\operatorname{div}\left(|\nabla u|^{p-2} \nabla u\right)=f \quad \text { in } \Omega,
$$

where $p>1$ and $\Omega$ is an open subset of $\mathbb{R}^{n}$.

The involved nonlinear potentials are denoted by $\mathbf{V}_{\alpha, p} f$ for $p \in(1, \infty)$ and $\alpha \in$ $(0, n)$, and are defined, for a locally integrable function $f$ in $\mathbb{R}^{n}$, by

$$
\mathbf{V}_{\alpha, p} f(x)=\mathbf{I}_{\alpha}\left(\mathbf{I}_{\alpha}|f|\right)^{\frac{1}{p-1}}(x) \quad \text { for } x \in \mathbb{R}^{n} .
$$

These potentials extend the Riesz potentials, since, if $2 \alpha<n$, then $\mathbf{V}_{\alpha, 2} f=$ $c \mathbf{I}_{2 \alpha}|f|$ for a suitable constant $c=c(\alpha, n)$ and for every measurable function $f$. They were introduced some forty years ago by V. P. Havin-V. G. Maz'ya [20], and extensively investigated in the framework of nonlinear capacity theory. Contributions on this topic include D. R. Adams-N. Meyers [3], D. R. Adams [2], and L. I. Hedberg-T. Wolff [21]. A comprehensive account of the theory of nonlinear potentials can be found in [1]. 
In the present paper we establish a sharp estimate for the decreasing rearrangement of $\mathbf{V}_{\alpha, p} f$ in terms of the decreasing rearrangement of $f$, extending inequality (1.3) to the nonlinear setting (Theorem 2.1, Section 2). This enables us to reduce the problem of the boundedness of $\mathbf{V}_{\alpha, p} f$ between rearrangement invariant spaces to equivalent one-dimensional inequalities for (nonlinear) Hardy-type operators (Corollary 2.3, Section 2). The special important cases of Lorentz and Orlicz spaces are discussed in Theorems 3.1 and 3.2, respectively, Section 3. Let us emphasize that, in general, sharp bounds for $\mathbf{V}_{\alpha, p}$ cannot be just derived on iterating bounds for $\mathbf{I}_{\alpha}$, even for $p=2$ - see e.g. [37] in this connection.

Our main motivation for the study of the potentials $\mathbf{V}_{\alpha, p}$ is in view of applications to rearrangement estimates for local solutions $u$ to quasilinear equations modelled on (1.5), and for their gradient, in terms of the datum $f$. They are derived in Theorems 4.1 and 4.4, respectively, Section 4, and follow from a combination of our bounds for $\mathbf{V}_{\alpha, p}$ with fundamental potential estimates established by T. Kilpelainen-J. Maly [24] for $u$, and by G. Mingione [32] $(p=2)$ and F. DuzaarG. Mingione $[17,18](p \geq 2)$ for $|\nabla u|$. As a consequence, the local integrability theory for solutions to quasilinear elliptic equations with $p$-growth, and for their gradients, is reduced to one-dimensional Hardy-type inequalities (Corollaries 4.2 and 4.5, Section 4). The case of Lorentz and Orlicz norms is explicitly worked out (Theorems 4.7-4.10, Section 4). In particular, we show that $|\nabla u|$ is locally bounded, and hence that $u$ is locally Lipschitz continuous, when $f$ belongs to a limiting Lorentz space (Corollary 4.11).

The rearrangement estimates for $u$ and $|\nabla u|$ presented here complement other well-known results in the literature. Our results concerning $u$ can be regarded as the local version of those obtained by G. Talenti $[38,39]$, and, in a somewhat implicit form, by V. G. Maz'ya [27, 28], which hold for boundary value problems. In fact, the techniques employed in these papers have a completely different nature. Related local estimates for the distribution function of solutions $u$ to Dirichlet problems, and ensuing bounds for Lorentz norms, are contained in [31].

As far as rearrangement estimates for $|\nabla u|$ are concerned, in the present paper we deal with equations involving smooth differential operators. As a consequence, we are able to discuss integrability properties of $|\nabla u|$ of any degree, including boundedness. By contrast, the rearrangement estimates for $|\nabla u|$ available in the literature $[5,6]$ only make use of coercivity assumptions on the differential operators. Therefore, they cannot lead to any bound for $|\nabla u|$ stronger than the natural energy associated with the equations. Moreover, they again apply to solutions to boundary value problems, but not to local solutions.

Part of the results of this paper were announced in [15].

AdDED to THE REVISED VERSION. We have been informed by G. Mingione that he and F. Duzaar have recently proved in [19] that the assumption $f \in L_{\text {loc }}^{n, \frac{1}{p-1}}(\Omega)$, which by Corollary 4.11 implies the Lipschitz continuity of solutions $u$ to (4.1), ensures that any such $u$ is in fact continuously differentiable. 


\section{Estimates for potentials}

Our rearrangement inequality between $\mathbf{V}_{\alpha, p} f$ and $f$ reads as follows.

Theorem 2.1. Let $p>1, n \geq 2$, and $0<\alpha<\frac{n}{p}$. Then there exist constants $C=C(\alpha, p, n)$ and $k=k(\alpha, p, n)$ such that

$$
\left(\mathbf{V}_{\alpha, p} f\right)^{*}(s) \leq C \int_{k s}^{\infty} r^{-1+\frac{\alpha p^{\prime}}{n}} f^{* *}(r)^{\frac{1}{p-1}} d r \quad \text { for } s>0,
$$

for every measurable function $f$ in $\mathbb{R}^{n}$ fulfilling (1.4). Here $p^{\prime}=\frac{p}{p-1}$. The result is sharp, in the sense that for every $k>0$ there exists a positive constant $C^{\prime}=$ $C^{\prime}(\alpha, p, n, k)$ such that, if $f$ is nonnegative and radially decreasing, then

$$
\left(\mathbf{V}_{\alpha, p} f\right)^{*}(s) \geq C^{\prime} \int_{k s}^{\infty} r^{-1+\frac{\alpha p^{\prime}}{n}} f^{* *}(r)^{\frac{1}{p-1}} d r \quad \text { for } s>0 .
$$

Remark 2.2. It will be clear from the proof of Theorem 2.1 that the right-hand side of (2.1) (and (2.2)), involving a double integral operator applied to $f^{*}$, is in fact equivalent to the sum of two single integral operators applied to $f^{*}$. Precisely, one has that

$$
\begin{aligned}
\int_{k s}^{\infty} r^{-1+\frac{\alpha p^{\prime}}{n}} f^{* *}(r)^{\frac{1}{p-1}} d r \approx & {\left[\left(s^{-1+\frac{\alpha p}{n}} \int_{0}^{k s} f^{*}(r) d r\right)^{\frac{1}{p-1}}\right.} \\
& \left.+\int_{k s}^{\infty} r^{-1+\frac{\alpha p^{\prime}}{n}} f^{*}(r)^{\frac{1}{p-1}} d r\right] \quad \text { for } s>0 .
\end{aligned}
$$

Here, $\approx$ means that the two sides of (2.3) can be estimated by each other up to multiplicative constants depending only on $\alpha, p$ and $n$. The expression on the righthand side of (2.3) can be easier to handle in some applications of Theorem 2.1.

By Theorem 2.1, the boundedness of the operator $\mathbf{V}_{\alpha, p}$ between arbitrary rearrangement invariant quasi-normed function spaces on measurable subsets $\Omega$ of $\mathbb{R}^{n}$ is reduced to more elementary inequalities for one-dimensional Hardy type operators in their representation spaces. Let us briefly recall a few definitions in this connection.

A quasi-normed function space $X(\Omega)$ on a measurable subset $\Omega$ of $\mathbb{R}^{n}$ is a linear space of measurable functions on $\Omega$ equipped with a functional $\|\cdot\|_{X(\Omega)}$, a quasi-norm, enjoying the following properties:

(i) $\|f\|_{X(\Omega)}>0$ if $f \neq 0$;

$\|\lambda f\|_{X(\Omega)}=|\lambda|\|f\|_{X(\Omega)}$ for every $\lambda \in \mathbb{R}$ and $f \in X(\Omega)$;

$\|f+g\|_{X(\Omega)} \leq c\left(\|f\|_{X(\Omega)}+\|g\|_{X(\Omega)}\right)$ for some constant $c \geq 1$ and for every $f, g \in X(\Omega)$

(ii) $\quad 0 \leq|g| \leq|f|$ a.e. in $\Omega$ implies $\|g\|_{X(\Omega)} \leq\|f\|_{X(\Omega)}$; 
(iii) $\quad 0 \leq f_{k} \nearrow f$ a.e. implies $\left\|f_{k}\right\|_{X(\Omega)} \nearrow\|f\|_{X(\Omega)}$ as $k \rightarrow \infty$;

(iv) if $G$ is a measurable subset of $\Omega$ and $|G|<\infty$, then $\left\|\chi_{G}\right\|_{X(\Omega)}<\infty$;

(v) for every measurable subset $G$ of $\Omega$ with $|G|<\infty$, there exists a constant $C$ such that $\int_{G}|f| d x \leq C\|f\|_{X(\Omega)}$ for every $f \in X(\Omega)$.

Given a measurable subset $G$ of $\Omega$, we denote by $\chi_{G}$ the characteristic function of $G$, and we define

$$
\|f\|_{X(G)}=\left\|f \chi_{G}\right\|_{X(\Omega)}
$$

for any measurable function $f$ on $\Omega$.

Moreover, we denote by $X_{\text {loc }}(\Omega)$ the space of measurable functions $f$ in $\Omega$ such that $\|f\|_{X(G)}<\infty$ for every compact set $G \subset \Omega$.

The space $X(\Omega)$ is called a Banach function space if (i) holds with $c=1$. In this case, the functional $\|\cdot\|_{X(\Omega)}$ is actually a norm which renders $X(\Omega)$ a Banach space.

A quasi-normed function space (in particular, a Banach function space) $X(\Omega)$ is called rearrangement invariant if there exists a quasi-normed function space $\bar{X}(0,|\Omega|)$ on the interval $(0,|\Omega|)$, called the representation space of $X(\Omega)$, having the property that

$$
\|f\|_{X(\Omega)}=\left\|f^{*}\right\|_{\bar{X}(0,|\Omega|)}
$$

for every $f \in X(\Omega)$. Here, and in what follows, any function $f$ on $\Omega$ is understood to be continued by 0 outside $\Omega$. Obviously, if $X(\Omega)$ is a rearrangement invariant quasi-normed space, then

$$
\|f\|_{X(\Omega)}=\|g\|_{X(\Omega)} \quad \text { if } \quad f^{*}=g^{*} .
$$

Note that, for customary spaces $X(\Omega)$, an expression for the quasi-norm $\|\cdot\|_{\bar{X}(0,|\Omega|)}$ is immediately derived from that of $\|\cdot\|_{X(\Omega)}$, via elementary properties of rearrangements. We refer the reader to [7] for a coprehensive treatment of rearrangement invariant spaces.

Theorem 2.3. Let $\alpha, p$ and $n$ be as in Theorem 2.1. Let $\Omega$ be a measurable subset of $\mathbb{R}^{n}$. Assume that either $\Omega=\mathbb{R}^{n}$ or $|\Omega|<\infty$. Let $X(\Omega)$ and $Y(\Omega)$ be quasi-normed rearrangement invariant spaces. Then the following assertions are equivalent.

(i) There exists a constant $C_{1}$ such that

$$
\left\|\left(\mathbf{V}_{\alpha, p} f\right)^{p-1}\right\|_{Y(\Omega)} \leq C_{1}\|f\|_{X(\Omega)}
$$

for every $f \in X(\Omega)$.

(ii) For every $k>0$, there exists a constant $C_{2}$ such that

$$
\left\|\left(\int_{k s}^{|\Omega|} r^{\left(-1+\frac{\alpha}{n}\right) p^{\prime}}\left(\int_{0}^{r} \varphi(\rho) d \rho\right)^{\frac{1}{p-1}} d r\right)^{p-1}\right\|_{\bar{Y}(0,|\Omega|)} \leq C_{2}\|\varphi\|_{\bar{X}(0,|\Omega|)}
$$

for every nonnegative function $\varphi \in \bar{X}(0,|\Omega|)$. 
(iii) For every $k>0$, there exists a constant $C_{3}$ such that

$$
\left\|s^{-1+\frac{\alpha p}{n}} \int_{0}^{k s} \varphi(r) d r\right\|_{\bar{Y}(0,|\Omega|)} \leq C_{3}\|\varphi\|_{\bar{X}(0,|\Omega|)}
$$

and

$$
\left\|\left(\int_{k s}^{|\Omega|} r^{-1+\frac{\alpha p^{\prime}}{n}} \varphi(r)^{\frac{1}{p-1}} d r\right)^{p-1}\right\|_{\bar{Y}(0,|\Omega|)} \leq C_{3}\|\varphi\|_{\bar{X}(0,|\Omega|)}
$$

for every nonnegative non-increasing function $\varphi \in \bar{X}(0,|\Omega|)$.

The derivation of Theorem 2.3 from Theorem 2.1 is straightforward when $\Omega=$ $\mathbb{R}^{n}$. In this connection, let us just notice that the admissible functions $\varphi$ in (ii) are not necessarily non-increasing, since $\int_{0}^{r} \varphi(\rho) d \rho \leq \int_{0}^{r} \varphi^{*}(\rho) d \rho$ for $r \geq 0$, by the Hardy-Littlewood inequality [7, Chapter 2, Theorem 2.2]. The case when $|\Omega|<\infty$ requires some additional (not difficult) consideration along the same lines as in the proof of [14, Theorems 1.3 and 1.4]. We omit the details for brevity.

Remark 2.4. When $X(\Omega)$ is a rearrangement invariant Banach function space, the constant $k$ can be set equal to 1 (or to any other positive value) in (2.7), (2.8) and (2.9). This is due to the fact that the dilation operator $D_{\delta}: \bar{X}(0,|\Omega|) \rightarrow \bar{X}(0,|\Omega|)$ defined for $\delta>0$ and $\varphi \in \bar{X}(0,|\Omega|)$ as

$$
D_{\delta} \varphi(s)= \begin{cases}\varphi(s \delta) & \text { if } s \delta \in(0,|\Omega|) \\ 0 & \text { otherwise }\end{cases}
$$

is bounded for any rearrangement invariant Banach function space $X(\Omega)$ [7, Chapter 3, Proposition 5.11].

Remark 2.5. Inequality (2.7) implies (2.6) for any measurable set $\Omega \subset \mathbb{R}^{n}$, namely even if $|\Omega|=\infty$ and $\Omega \neq \mathbb{R}^{n}$. Analogously, inequalities (2.8)-(2.9) imply (2.6) for any $\Omega \subset \mathbb{R}^{n}$.

Inequality (2.7) is stronger, in general, than (2.9), since, if $\varphi:(0,|\Omega|) \rightarrow$ $[0, \infty)$ is non-increasing, then

$$
\varphi(s) \leq \frac{1}{s} \int_{0}^{s} \varphi(r) d r \quad \text { for } s>0 .
$$

However, in the special case when the quasi-norm in $X(\Omega)$ fulfils

$$
\left\|\frac{1}{s} \int_{0}^{s} \varphi(r) d r\right\|_{\bar{X}(0,|\Omega|)} \leq C\|\varphi\|_{\bar{X}(0,|\Omega|)}
$$

for some constant $C$ and for every $\varphi \in \bar{X}(0,|\Omega|)$, inequalities (2.7) and (2.9) turn out to be equivalent. As a consequence, we have the following corollary. 
Corollary 2.6. Let $\alpha, p, n, \Omega, X(\Omega)$ and $Y(\Omega)$ be as in Theorem 2.3. Assume, in addition, that $X(\Omega)$ fulfils (2.11). Then (2.6) is equivalent to (2.9).

Note that the rearrangement invariant Banach function spaces $X(\Omega)$ making inequality (2.11) true can be characterized in terms of their upper Boyd index $I(X) \in[0,1]$, defined by

$$
I(X)=\lim _{\delta \rightarrow 0} \frac{\log \left\|D_{\delta}\right\|}{\log (1 / \delta)},
$$

where $\left\|D_{\delta}\right\|$ is the norm of $D_{\delta}$. Indeed, inequality (2.11) holds if and only if $I(X)<$ 1 [7, Theorem 5.15].

Our approach to Theorem 2.1 relies upon results from interpolation theory, and is related to arguments from $[16,23]$. We begin by recalling a few facts from this theory to be used in what follows.

Let $\left(X_{1}(\Omega), X_{2}(\Omega)\right)$ be a couple of quasi-normed function spaces. Their $K-$ functional is defined for $f \in X_{1}(\Omega)+X_{2}(\Omega)$ as

$$
K\left(s, f ; X_{1}, X_{2}\right)=\inf _{f=f_{1}+f_{2}}\left(\left\|f_{1}\right\|_{X_{1}(\Omega)}+s\left\|f_{2}\right\|_{X_{2}(\Omega)}\right) \quad \text { for } s>0 .
$$

An operator $T$ defined on a linear space of measurable functions on $\Omega$, and taking values into the space of measurable functions in $\Omega$, is called quasi-linear if there exists a constant $c$ such that, for every $f$ and $g$ in the domain of $T$ and every $\lambda \in \mathbb{R}$,

$$
|T(\lambda f)|=|\lambda||T f|,
$$

and

$$
|T(f+g)| \leq c(|T f|+|T g|) .
$$

The following result is basic in the theory of real interpolation for linear operators. We state and prove it in a slightly more general form needed for our purposes.

Proposition 2.7. Let $\Omega$ be a measurable subset of $\mathbb{R}^{n}$ and let $T$ be a quasi-linear operator defined on a linear space of measurable functions on $\Omega$. Let $X_{i}(\Omega)$ and $Y_{i}(\Omega), i=1,2$, be quasi-normed function spaces on $\Omega$. Assume that

$$
T: X_{i}(\Omega) \rightarrow Y_{i}(\Omega)
$$

with norms $N_{i}, i=1,2$, where the arrow " $\rightarrow$ " denotes a bounded operator. Then, for every $f \in X_{1}(\Omega)+X_{2}(\Omega)$,

$$
K\left(T f, s ; Y_{1}, Y_{2}\right) \leq c N_{1} K\left(f, s N_{2} / N_{1} ; X_{1}, X_{2}\right) \quad \text { for } s>0,
$$

where $c$ is the constant appearing in (2.13). 
Proof. We preliminarily observe that, if $h, g \in Y_{1}(\Omega)+Y_{2}(\Omega)$ and $|h| \leq|g|$ a.e. in $\Omega$, then

$$
K\left(h, s ; Y_{1}, Y_{2}\right) \leq K\left(g, s ; Y_{1}, Y_{2}\right) .
$$

To verify (2.16), consider any decomposition $g=g_{1}+g_{2}$ with $g_{i} \in Y_{i}(\Omega), i=1,2$, of $g$. Set $h_{i}=\frac{h}{g} g_{i}, i=1,2$. Then $h=h_{1}+h_{2}$ is a decomposition of $h$, and

$$
K\left(h, s ; Y_{1}, Y_{2}\right) \leq\left\|\frac{h}{g} g_{1}\right\|_{Y_{1}(\Omega)}+s\left\|\frac{h}{g} g_{2}\right\|_{Y_{2}(\Omega)} \leq\left\|g_{1}\right\|_{Y_{1}(\Omega)}+s\left\|g_{2}\right\|_{Y_{2}(\Omega)} .
$$

Inequality (2.16) is a consequence of (2.17) and of the arbitrariness of the decomposition of $g$. Now, let $f=f_{1}+f_{2}$, with $f_{i} \in X_{i}(\Omega), i=1,2$, be a decomposition of $f$. Owing to (2.13), $|T f| \leq c\left(\left|T f_{1}\right|+\left|T f_{2}\right|\right)$. Thus, by (2.16),

$$
\begin{aligned}
K\left(T f, s ; Y_{1}, Y_{2}\right) & \leq K\left(c\left|T f_{1}\right|+c\left|T f_{2}\right|, s ; Y_{1}, Y_{2}\right) \leq c\left\|T f_{1}\right\|_{Y_{1}(\Omega)}+c s\left\|T f_{2}\right\|_{Y_{2}(\Omega)} \\
& \leq c N_{1}\left\|f_{1}\right\|_{X_{1}(\Omega)}+c s N_{2}\left\|f_{2}\right\|_{X_{2}(\Omega)} \\
& =c N_{1}\left(\left\|f_{1}\right\|_{X_{1}(\Omega)}+s \frac{N_{2}}{N_{1}}\left\|f_{2}\right\|_{X_{2}(\Omega)}\right) \text { for } s>0 .
\end{aligned}
$$

Hence, inequality (2.15) follows, thanks to the arbitrariness of the decomposition of $f$.

Proposition 2.7 will be applied to the case when $T=\left(\mathbf{V}_{\alpha, p}\right)^{p-1}$, and the quasinormed function spaces $X_{i}(\Omega)$ and $Y_{i}(\Omega)$ are Lorentz spaces. Recall that, if either $\sigma \in(1, \infty]$ and $\varrho \in(0, \infty]$, or $\sigma=1$ and $\varrho \in(0,1]$, the Lorentz space $L^{\sigma, \varrho}(\Omega)$ is defined as the set of all measurable functions $f$ on $\Omega$ for which the expression

$$
\|f\|_{L^{\sigma, \varrho}(\Omega)}=\left\|s^{\frac{1}{\sigma}-\frac{1}{\varrho}} f^{*}(s)\right\|_{L^{\varrho}(0,|\Omega|)}
$$

is finite. In particular,

$$
L^{\sigma, \sigma}(\Omega)=L^{\sigma}(\Omega)
$$

for every $\sigma \in[1, \infty]$. Moreover, $L^{\sigma, \varrho_{1}}(\Omega) \varsubsetneqq L^{\sigma, \varrho_{2}}(\Omega)$ if $\varrho_{1}<\varrho_{2}$, and if $|\Omega|<\infty$, $L_{\text {loc }}^{\sigma_{1}, \varrho_{1}}(\Omega) \varsubsetneqq L_{\text {loc }}^{\sigma_{2}, \varrho_{2}}(\Omega)$ if $\sigma_{1}>\sigma_{2}$ and $\varrho_{1}, \varrho_{2}$ are admissible exponents in $(0, \infty]$.

If $\sigma>1$, then

$$
\left\|s^{\frac{1}{\sigma}-\frac{1}{\varrho}} f^{*}(s)\right\|_{L^{\varrho}(0,|\Omega|)} \approx\left\|s^{\frac{1}{\sigma}-\frac{1}{\varrho}} f^{* *}(s)\right\|_{L^{\varrho}(0,|\Omega|)},
$$

up to multiplicative constants depending on $\sigma$ and $\varrho$. Thus, in particular, the space $L^{\sigma, \varrho}(\Omega)$ fulfils (2.11) when $\sigma>1$. Moreover, if either $\sigma>1$ and $\varrho \in[1, \infty]$, or $\sigma=\varrho=1$, then $L^{\sigma, \varrho}(\Omega)$ is in fact a Banach function space, up to equivalent norms.

The endpoint Lorentz spaces to be used in our application of the $K$-functional to $\left(\mathbf{V}_{\alpha, p}\right)^{p-1}$ are dictated by related endpoint boundedness properties of the linear Riesz potential $\mathbf{I}_{\alpha}$ in Lorentz spaces. The relevant properties are well-known when Lorentz spaces which are Banach function spaces are involved. In the following Proposition 2.8 the case of quasi-normed Lorentz spaces is included. 
Proposition 2.8. Let $0<\alpha<n$.

(i) If $1<\sigma<\frac{n}{\alpha}$ and $0<\varrho \leq \infty$, then

$$
\mathbf{I}_{\alpha}: L^{\sigma, \varrho}\left(\mathbb{R}^{n}\right) \rightarrow L^{\frac{\sigma n}{n-\alpha \sigma}, \varrho}\left(\mathbb{R}^{n}\right) ;
$$

(ii)

$$
\mathbf{I}_{\alpha}: L^{1}\left(\mathbb{R}^{n}\right) \rightarrow L^{\frac{n}{n-\alpha}, \infty}\left(\mathbb{R}^{n}\right)
$$

(iii)

$$
\mathbf{I}_{\alpha}: L^{\frac{n}{\alpha}, 1}\left(\mathbb{R}^{n}\right) \rightarrow L^{\infty}\left(\mathbb{R}^{n}\right) .
$$

Proof. By (1.3) and (2.19), Part (i) is reduced to the Hardy-type inequality

$$
\left\|s^{\frac{n-\alpha \sigma}{n \sigma}-\frac{1}{\varrho}} \int_{s}^{\infty} r^{-1+\frac{\alpha}{n}} f^{* *}(r) d r\right\|_{L^{\varrho}(0, \infty)} \leq C\left\|s^{\frac{1}{\sigma}-\frac{1}{\varrho}} f^{* *}(s)\right\|_{L^{\varrho}(0, \infty)}
$$

for some constant $C=C(\alpha, n, \sigma, \varrho)$ and for every $f \in L^{\sigma, \varrho}\left(\mathbb{R}^{n}\right)$. Inequality (2.23) follows via a classical weighted Hardy type inequality if $\varrho \geq 1$ ( [29, Chapter 1]), and via a weighted Hardy type inequality for non-increasing functions if $0<\varrho<1$ [10].

As for (ii), we have that

$$
\begin{aligned}
\left\|\mathbf{I}_{\alpha} f\right\|_{L^{\frac{n}{n-\alpha}, \infty}\left(\mathbb{R}^{n}\right)} & \leq C \sup _{s>0} s^{1-\frac{\alpha}{n}} \int_{s}^{\infty} r^{-1+\frac{\alpha}{n}} f^{* *}(r) d r \\
& \leq\|f\|_{L^{1}\left(\mathbb{R}^{n}\right)} \sup _{s>0} s^{1-\frac{\alpha}{n}} \int_{s}^{\infty} r^{-2+\frac{\alpha}{n}} d r=C^{\prime}\|f\|_{L^{1}\left(\mathbb{R}^{n}\right)}
\end{aligned}
$$

for some constants $C=C(\alpha, n)$ and $C^{\prime}=C^{\prime}(\alpha, n)$, and for every $f \in L^{1}\left(\mathbb{R}^{n}\right)$. Finally, Part (iii) is a consequence of the chain

$$
\begin{aligned}
\left\|\mathbf{I}_{\alpha} f\right\|_{L^{\infty}\left(\mathbb{R}^{n}\right)} & \leq C \int_{0}^{\infty} r^{-1+\frac{\alpha}{n}} f^{* *}(r) d r \\
& =\int_{0}^{\infty} f^{*}(\rho) \int_{\rho}^{\infty} r^{-2+\frac{\alpha}{n}} d r d \rho=C^{\prime}\|f\|_{L^{\frac{n}{\alpha}, 1}\left(\mathbb{R}^{n}\right)}
\end{aligned}
$$

for some constants $C=C(\alpha, n)$ and $C^{\prime}=C^{\prime}(\alpha, n)$, and for every $f \in L^{\frac{n}{\alpha}, 1}\left(\mathbb{R}^{n}\right)$.

Our last preliminary result is a characterization of the space $L^{1}\left(\mathbb{R}^{n}\right)+L^{\frac{n}{\alpha p}, \frac{1}{p-1}}\left(\mathbb{R}^{n}\right)$.

Lemma 2.9. Let $\alpha$, $p$ and $n$ be as in Theorem 2.1. Then $f \in L^{1}\left(\mathbb{R}^{n}\right)+L^{\frac{n}{\alpha p}, \frac{1}{p-1}}\left(\mathbb{R}^{n}\right)$ if and only if

$$
\int_{0}^{1} f^{*}(s) d s+\int_{1}^{\infty} s^{-1+\frac{\alpha p^{\prime}}{n}} f^{*}(s)^{\frac{1}{p-1}} d s<\infty .
$$


Proof. Assume that (2.26) holds. Consider the decomposition $f=f_{1}+f_{2}$, where $f_{2}=\min \left\{|f|, f^{*}(1)\right\} \operatorname{sign}(f)$. Since $f_{2}^{*}(s)=\min \left\{f^{*}(s), f^{*}(1)\right\}$, we have that

$$
\left\|f_{2}\right\|_{L^{\frac{n}{\alpha p}, \frac{1}{p-1}}\left(\mathbb{R}^{n}\right)}^{\frac{1}{p-1}}=f^{*}(1)^{\frac{1}{p-1}} \int_{0}^{1} s^{-1+\frac{\alpha p^{\prime}}{n}} d s+\int_{1}^{\infty} s^{-1+\frac{\alpha p^{\prime}}{n}} f^{*}(s)^{\frac{1}{p-1}} d s<\infty .
$$

On the other hand,

$$
\left\|f_{1}\right\|_{L^{1}\left(\mathbb{R}^{n}\right)}=\int_{0}^{1} f^{*}(s) d s-f^{*}(1)<\infty
$$

Hence, $f \in L^{1}\left(\mathbb{R}^{n}\right)+L^{\frac{n}{\alpha p}, \frac{1}{p-1}}\left(\mathbb{R}^{n}\right)$.

Conversely, assume that $f \in L^{1}\left(\mathbb{R}^{n}\right)+L^{\frac{n}{\alpha p}, \frac{1}{p-1}}\left(\mathbb{R}^{n}\right)$. Hence, $f=f_{1}+f_{2}$ with $f_{1} \in L^{1}\left(\mathbb{R}^{n}\right)$ and $f_{2} \in L^{\frac{n}{\alpha p}, \frac{1}{p-1}}\left(\mathbb{R}^{n}\right)$. The former membership implies that

$$
\int_{0}^{1} f_{1}^{*}(s) d s<\infty
$$

Also,

$$
\int_{0}^{1} f_{2}^{*}(s) d s<\infty
$$

by property (v) of the definition of quasi-normed space, since $\left|\left\{f_{2}>f_{2}^{*}(1)\right\}\right|<\infty$. On the other hand,

$$
\int_{1}^{\infty} s^{-1+\frac{\alpha p^{\prime}}{n}} f_{2}^{*}(s)^{\frac{1}{p-1}} d s<\infty
$$

inasmuch as $f_{2} \in L^{\frac{n}{\alpha p}, \frac{1}{p-1}}\left(\mathbb{R}^{n}\right)$. Moreover, since $\int_{1}^{\infty} f_{1}^{*}(s)<\infty$ and $f_{1}^{*}$ is nonincreasing, there exists a constant $c$ such that $f_{1}^{*}(s) \leq \frac{c}{s}$ for $s \geq 1$. Consequently,

$$
\int_{1}^{\infty} s^{-1+\frac{\alpha p^{\prime}}{n}} f_{1}^{*}(s)^{\frac{1}{p-1}} d s<\infty
$$

since $\alpha<\frac{n}{p}$. A property of rearrangements implies that $\left(f_{1}+f_{2}\right)^{*}(s) \leq f_{1}^{*}(s / 2)+$ $f_{2}^{*}(s / 2)$ for $s>0$. Hence, (2.26) follows via (2.27)-(2.30).

We are now in a position to prove Theorem 2.1 .

Proof of Theorem 2.1. The operator $\left(\mathbf{V}_{\alpha, p}\right)^{p-1}$ is clearly quasi-linear, with constant $c$ in (2.13) depending on $p$. We claim that

$$
\left(\mathbf{V}_{\alpha, p}\right)^{p-1}: L^{1}\left(\mathbb{R}^{n}\right) \rightarrow L^{\frac{n}{n-\alpha p}, \infty}\left(\mathbb{R}^{n}\right)
$$

and

$$
\left(\mathbf{V}_{\alpha, p}\right)^{p-1}: L^{\frac{n}{\alpha p}, \frac{1}{p-1}}\left(\mathbb{R}^{n}\right) \rightarrow L^{\infty}\left(\mathbb{R}^{n}\right)
$$


Indeed, there exist constants $C=C(\alpha, p, n)$ and $C^{\prime}=C^{\prime}(\alpha, p, n)$ such that

$$
\begin{aligned}
\left\|\left(\mathbf{V}_{\alpha, p} f\right)^{p-1}\right\|_{L^{\frac{n}{n-\alpha p}, \infty}\left(\mathbb{R}^{n}\right)} & =\left\|\mathbf{I}_{\alpha}\left(\mathbf{I}_{\alpha}|f|\right)^{\frac{1}{p-1}}\right\|_{L^{\frac{n(p-1)}{n-\alpha p}, \infty}}^{p-1}\left(\mathbb{R}^{n}\right) \\
& \leq C\left\|\left(\mathbf{I}_{\alpha}|f|\right)^{\frac{1}{p-1}}\right\|_{L^{\frac{n(p-1)}{n-\alpha}, \infty}\left(\mathbb{R}^{n}\right)}^{p-1} \\
& =C\left\|\mathbf{I}_{\alpha}|f|\right\|_{L^{\frac{n}{n-\alpha}, \infty}\left(\mathbb{R}^{n}\right)} \leq C^{\prime}\|f\|_{L^{1}\left(\mathbb{R}^{n}\right)}
\end{aligned}
$$

for every $f \in L^{1}\left(\mathbb{R}^{n}\right)$, where the first inequality holds by (2.20) and the last one by (2.21). Hence, (2.31) follows. As for (2.32), it is a consequence of the following chain:

$$
\begin{aligned}
\left\|\left(\mathbf{V}_{\alpha, p} f\right)^{p-1}\right\|_{L^{\infty}\left(\mathbb{R}^{n}\right)} & =\left\|\mathbf{I}_{\alpha}\left(\mathbf{I}_{\alpha}|f|\right)^{\frac{1}{p-1}}\right\|_{L^{\infty}\left(\mathbb{R}^{n}\right)}^{p-1} \\
& \leq C\left\|\left(\mathbf{I}_{\alpha}|f|\right)^{\frac{1}{p-1}}\right\|_{L^{\frac{n}{\alpha}, 1}\left(\mathbb{R}^{n}\right)}^{p-1} \\
& =C\left\|\mathbf{I}_{\alpha}|f|\right\|{ }_{L^{\frac{n}{\alpha(p-1)}}, \frac{1}{p-1}}\left(\mathbb{R}^{n}\right) \\
& \leq C^{\prime}\|f\|_{L^{\frac{n}{\alpha p}, \frac{1}{p-1}}\left(\mathbb{R}^{n}\right)},
\end{aligned}
$$

which holds for some constants $C=C(\alpha, p, n)$ and $C^{\prime}=C^{\prime}(\alpha, p, n)$ and for every $f \in L^{\frac{n}{\alpha p}, \frac{1}{p-1}}\left(\mathbb{R}^{n}\right)$. Note that the first inequality holds in (2.34) owing to (2.22), whereas the second one is due to (2.20). The idea is now to exploit Proposition 2.7 and derive the rearrangement estimate (2.1) from (2.31) and (2.32). To this purpose, we need expressions for the K-functionals of the couples $\left(L^{1}\left(\mathbb{R}^{n}\right), L^{\frac{n}{\alpha p}, \frac{1}{p-1}}\left(\mathbb{R}^{n}\right)\right)$ and $\left(L^{\frac{n}{n-\alpha p}, \infty}\left(\mathbb{R}^{n}\right), L^{\infty}\left(\mathbb{R}^{n}\right)\right)$. These follow (up to multiplicative constants) from results of [22]. Actually, [22, Theorem 4.2] tells us that, if $f \in L^{1}\left(\mathbb{R}^{n}\right)+L^{\frac{n}{\alpha p}, \frac{1}{p-1}}\left(\mathbb{R}^{n}\right)$, then

$$
\begin{aligned}
& K\left(f, s ; L^{1}\left(\mathbb{R}^{n}\right), L^{\frac{n}{\alpha p}, \frac{1}{p-1}}\left(\mathbb{R}^{n}\right)\right) \\
& \approx \int_{0}^{s^{\frac{n}{n-\alpha p}}} f^{*}(r) d r+s\left(\int_{s^{\frac{n}{n-\alpha p}}}^{\infty} r^{-1+\frac{\alpha p^{\prime}}{n}} f^{*}(r)^{\frac{1}{p-1}} d r\right)^{p-1} \quad \text { for } s>0,
\end{aligned}
$$

with equivalence constants depending on $\alpha, p$ and $n$. Moreover, by [22, Equation (4.8)], we have that, if $g \in L^{\frac{n}{n-\alpha p}, \infty}\left(\mathbb{R}^{n}\right)+L^{\infty}\left(\mathbb{R}^{n}\right)$, then

$$
K\left(g, s ; L^{\frac{n}{n-\alpha p}, \infty}\left(\mathbb{R}^{n}\right), L^{\infty}\left(\mathbb{R}^{n}\right)\right) \approx\left\|r^{1-\frac{\alpha p}{n}} g^{*}(r)\right\|_{L^{\infty}\left(0, s^{\frac{n}{n-\alpha p}}\right)} \quad \text { for } s>0,
$$

with equivalence constants depending on $\alpha, p$ and $n$. 
If $f \in L^{1}\left(\mathbb{R}^{n}\right)+L^{\frac{n}{\alpha p} \frac{1}{p-1}}\left(\mathbb{R}^{n}\right)$, then by Proposition 2.7 and equations (2.35) and (2.36) we deduce that a constant $C=C(\alpha, p, n)$ exists such that

$$
\begin{aligned}
& s^{1-\frac{\alpha p}{n}}\left(\mathbf{V}_{\alpha, p} f\right)^{*}(s) \leq\left\|r^{1-\frac{\alpha p}{n}}\left(\mathbf{V}_{\alpha, p} f\right)^{*}(r)\right\|_{L^{\infty}(0, s)} \\
& \leq C\left[N_{1} \int_{0}^{k s} f^{*}(r) d r+N_{2} s^{1-\frac{\alpha p}{n}}\left(\int_{k s}^{\infty} r^{-1+\frac{\alpha p^{\prime}}{n}} f^{*}(r)^{\frac{1}{p-1}} d r\right)^{p-1}\right] \text { for } s>0,
\end{aligned}
$$

where $k=\left(N_{2} / N_{1}\right)^{\frac{n}{n-\alpha p}}$. Note that

$$
\begin{aligned}
& \left(\int_{s}^{\infty}\left(r^{\left(-1+\frac{\alpha}{n}\right) p} \int_{0}^{r} f^{*}(\rho) d \rho\right)^{\frac{1}{p-1}} d r\right)^{p-1} \\
& \geq\left(\int_{0}^{s} f^{*}(\rho) d \rho\right)\left(\int_{s}^{\infty} r^{\left(-1+\frac{\alpha}{n}\right) p^{\prime}} d r\right)^{p-1} \\
& =C s^{-1+\frac{\alpha p}{n}} \int_{0}^{s} f^{*}(\rho) d \rho \quad \text { for } s>0
\end{aligned}
$$

for some positive constant $C=C(\alpha, p, n)$. Also, since $f^{*}$ is non-increasing, by (2.10)

$$
\begin{aligned}
& \left(\int_{s}^{\infty}\left(r^{\left(-1+\frac{\alpha}{n}\right) p} \int_{0}^{r} f^{*}(\rho) d \rho\right)^{\frac{1}{p-1}} d r\right)^{p-1} \\
& \geq\left(\int_{s}^{\infty} r^{-1+\frac{\alpha p^{\prime}}{n}} f^{*}(r)^{\frac{1}{p-1}} d r\right)^{p-1} \text { for } s>0 .
\end{aligned}
$$

Inequality (2.1) follows from (2.37)-(2.39).

In the case when $f \notin L^{1}\left(\mathbb{R}^{n}\right)+L^{\frac{n}{\alpha p}, \frac{1}{p-1}}\left(\mathbb{R}^{n}\right)$, by Lemma 2.9 and (2.38)-(2.39), the right-hand side of (2.1) is identically infinite, and hence (2.1) holds trivially.

We next establish the lower bound (2.2) for functions $f$ having the form $f(x)=$ $f^{*}\left(\omega_{n}|x|^{n}\right)$ for $x \in \mathbb{R}^{n}$. Here, $\omega_{n}$ denotes the Lebesgue norm of the unit ball in $\mathbb{R}^{n}$. We assume that $k=1$, the general case being analogous. Since $|x-y| \leq|x|+|y|$ for $x, y \in \mathbb{R}^{n}$, one has that

$$
\begin{aligned}
& \mathbf{I}_{\alpha} f(x) \geq 2^{\alpha-n}\left(\int_{|y|<|x|} \frac{f(y)}{|x|^{n-\alpha}} d y+\int_{|y| \geq|x|} \frac{f(y)}{|y|^{n-\alpha}} d y\right) \\
& =\omega_{n}^{1-\frac{\alpha}{n}} 2^{\alpha-n}\left(|x|^{\alpha-n} \int_{0}^{\omega_{n}|x|^{n}} f^{*}(s) d s+\int_{\omega_{n}|x|^{n}}^{\infty} s^{-1+\frac{\alpha}{n}} f^{*}(s) d s\right) \text { for } x \in \mathbb{R}^{n} .
\end{aligned}
$$


Since the rightmost side of (2.40) is a radially decreasing function, an iteration of this estimate yields

$$
\begin{aligned}
\mathbf{V}_{\alpha, p} f(x) \geq & C\left[|x|^{\alpha-n} \int_{0}^{\omega_{n}|x|^{n}}\left(r^{-1+\frac{\alpha}{n}} \int_{0}^{r} f^{*}(\rho) d \rho+\int_{r}^{\infty} \rho^{-1+\frac{\alpha}{n}} f^{*}(\rho) d \rho\right)^{\frac{1}{p-1}} d r\right. \\
& \left.+\int_{\omega_{n}|x|^{n}}^{\infty} r^{-1+\frac{\alpha}{n}}\left(r^{-1+\frac{\alpha}{n}} \int_{0}^{r} f^{*}(\rho) d \rho+\int_{r}^{\infty} \rho^{-1+\frac{\alpha}{n}} f^{*}(\rho) d \rho\right)^{\frac{1}{p-1}} d r\right] \\
\geq & C \int_{\omega_{n}|x|^{n}}^{\infty}\left(r^{\left(-1+\frac{\alpha}{n}\right) p} \int_{0}^{r} f^{*}(\rho) d \rho\right)^{\frac{1}{p-1}} d r \text { for } x \in \mathbb{R}^{n},
\end{aligned}
$$

for some positive constant $C=C(\alpha, p, n)$. Hence (2.2) follows.

\section{Boundedness of potentials in Orlicz and Lorentz spaces}

The Lebesgue spaces are probably the most classical instance of rearrangement invariant spaces. The Lorentz spaces and the Orlicz spaces provide generalizations of the Lebesgue spaces in different directions. In the present section we discuss boundedness properties of nonlinear potentials in these spaces.

We begin with the Lorentz spaces. Besides the standard Lorentz spaces, whose definition is recalled in Section 2, the Lorentz-Zygmund spaces, a further generalization of them, will come into play in certain borderline situations.

If $|\Omega|<\infty$, and either $\sigma \in(1, \infty], \varrho \in(0, \infty], \beta \in \mathbb{R}$, or $\sigma=1, \varrho \in(0,1]$, $\beta \in[0, \infty)$, the Lorentz-Zygmund space $L^{\sigma, \varrho}(\log L)^{\beta}(\Omega)$ is defined as the set of all measurable functions $f$ on $\Omega$ making the expression

$$
\|f\|_{L^{\sigma, \varrho}(\log L)^{\beta}(\Omega)}=\left\|s^{\frac{1}{\sigma}-\frac{1}{\varrho}}(1+\log (|\Omega| / s))^{\beta} f^{*}(s)\right\|_{L^{\varrho}(0,|\Omega|)}
$$

finite. If $\varrho \geq 1$ and the weight multiplying $f^{*}(s)$ on the right-hand side of (3.1) is non-increasing, then the functional $\|f\|_{L^{\sigma, \varrho}(\log L)^{\beta}(\Omega)}$ is actually a norm, and $L^{\sigma, \varrho}(\log L)^{\beta}(\Omega)$ is a rearrangement invariant Banach function space equipped with this norm. Otherwise, this functional is only a quasi-norm. For certain values of the parameters $\sigma, \varrho$ and $\beta$, it is however equivalent to a rearrangement invariant norm obtained on replacing $f^{*}$ by $f^{* *}$ in the definition, up to equivalent constants. We refer to [34] for a comprehensive picture on this topic.

Theorem 3.1. Let $\alpha, p$ and $n$ be as in Theorem 2.1. Let $\Omega$ be a measurable subset of $\mathbb{R}^{n}$.

(i) If $0<\varrho \leq \infty$ and $1<\sigma<\frac{n}{\alpha p}$, then there exists a constant $C=C(\alpha, p, n, \sigma, \varrho)$ such that

$$
\left\|\mathbf{V}_{\alpha, p} f\right\|_{L^{\frac{\sigma n(p-1)}{n-\sigma \alpha p}, \varrho(p-1)}(\Omega)} \leq C\|f\|_{L^{\sigma, \varrho}(\Omega)}^{\frac{1}{p-1}}
$$

for every $f \in L^{\sigma, \varrho}(\Omega)$. 
(ii) If $\sigma=1$ and $0<\varrho \leq 1$, then there exists a constant $C=C(\alpha, p, n, \varrho)$ such that

$$
\left\|\mathbf{V}_{\alpha, p} f\right\|_{L^{\frac{n(p-1)}{n-\alpha p}, \infty}(\Omega)} \leq C\|f\|_{L^{1, \varrho}(\Omega)}^{\frac{1}{p-1}}
$$

for every $f \in L^{1, \varrho}(\Omega)$.

(iii) If $|\Omega|<\infty$ and $\varrho>\frac{1}{p-1}$, then there exists a constant $C=C(\alpha, p, n, \varrho,|\Omega|)$ such that

$$
\left\|\mathbf{V}_{\alpha, p} f\right\|_{L^{\infty, \varrho(p-1)}(\log L)^{-1}(\Omega)} \leq C\|f\|_{L^{\frac{1}{\alpha p}, \varrho}(\Omega)}^{\frac{\frac{1}{p-1}}{n^{2}}}
$$

for every $f \in L^{\frac{n}{\alpha p}, \varrho}(\Omega)$.

(iv) If $\sigma=\frac{n}{\alpha p}$ and $\varrho \leq \frac{1}{p-1}$, then there exists a constant $C=C(\alpha, n, p)$ such that

$$
\left\|\mathbf{V}_{\alpha, p} f\right\|_{L^{\infty}(\Omega)} \leq C\|f\|_{L^{\frac{n}{\alpha p}, \varrho}(\Omega)}^{\frac{1}{p-1}}
$$

for every $f \in L^{\frac{n}{\alpha p}, \varrho}(\Omega)$.

Part (i) of Theorem 3.1 follows quite easily from Theorem 2.3, Corollary 2.6 and Remark 2.5, owing to a classical weighted Hardy type inequality ([29, Chapter 1]) if $\varrho \geq \frac{1}{p-1}$, and to a weighted Hardy type inequality for non-increasing functions if $0<\varrho<\frac{1}{p-1}$ ([10]). The proof of Part (iii) also relies upon a classical weighted Hardy inequality. Parts (ii) and (iv) can be derived from an analogous argument as in the proof of Proposition 2.8. We omit the details for brevity.

We now focus on the boundedness of $\mathbf{V}_{\alpha, p}$, or more precisely of $\left(\mathbf{V}_{\alpha, p}\right)^{p-1}$, in Orlicz spaces. A Young function $A:[0, \infty) \rightarrow[0, \infty]$ is a convex function, vanishing at 0 , which is neither identically equal to 0 , nor to $\infty$. With any Young function $A$ it is associated the Orlicz space $L^{A}(\Omega)$, namely the rearrangement invariant space of those measurable functions $f$ on $\Omega$ such that the Luxemburg norm

$$
\|f\|_{L^{A}(\Omega)}=\inf \left\{\lambda>0: \int_{\Omega} A\left(\frac{|f(x)|}{\lambda}\right) d x \leq 1\right\}
$$

is finite.

Two Young functions $A$ and $B$ are said to be equivalent near infinity if positive constants $c_{1}, c_{2}$ and $t_{0}$ exist such that

$$
A\left(c_{1} t\right) \leq B(t) \leq A\left(c_{2} t\right) \quad \text { for } t \geq t_{0}
$$

If $|\Omega|<\infty$, then

$$
\begin{array}{r}
L^{A}(\Omega)=L^{B}(\Omega) \quad(\text { up to equivalent norms }) \text { if and only if } \\
A \text { and } B \text { are equivalent near infinity. }
\end{array}
$$


Let $A$ and $B$ be Young functions, and let $0<\alpha<\frac{n}{p}$. We define the functions $E_{\alpha, p}$ and $F_{\alpha, p}:[0, \infty) \rightarrow[0, \infty]$ as

$$
E_{\alpha, p}(t)= \begin{cases}\left(\int_{0}^{t}\left(\frac{\tau^{\frac{1}{p-1}-1+\frac{\alpha p^{\prime}}{n}}}{A(\tau)^{\frac{\alpha p^{\prime}}{n}}}\right)^{\frac{n}{n-\alpha p^{\prime}}} d \tau\right)^{p-1-\frac{\alpha p}{n}} & \text { if } \alpha p^{\prime}<n, \\ \sup _{\tau \in(0, t)} \frac{\tau}{A(\tau)^{\frac{\alpha p}{n}}} & \text { if } \alpha p^{\prime} \geq n,\end{cases}
$$

and

$$
F_{\alpha, p}(t)=\left(\int_{0}^{t} \frac{B(\tau)}{\tau^{1+\frac{n}{n-\alpha p}}} d \tau\right)^{\frac{n-\alpha p}{n}}
$$

for $t \geq 0$. Our discussion of the boundedness properties of the operator $\left(\mathbf{V}_{\alpha, p}\right)^{p-1}$ in Orlicz spaces involves the functions $E_{\alpha, p}$ and $F_{\alpha, p}$, and is the content of the next theorem. Analogous results for $\mathbf{I}_{\alpha}$ can be found in [12] (see also [13] for an alternate approach).

Theorem 3.2. Let $\alpha, p$ and $n$ be as in Theorem 2.1. Let $\Omega$ be a measurable subset of $\mathbb{R}^{n}$. Assume that $A$ and $B$ are Young functions such that the function $E_{\alpha, p}$ defined by (3.8) is finite-valued, and there exists $\gamma>0$ such that

$$
F_{\alpha, p}\left(\frac{E_{\alpha, p}(t)}{\gamma}\right) \leq \gamma \frac{A(t)}{t} \quad \text { for } t>0 .
$$

Then there exists a constant $C=C(\alpha, p, n, \gamma)$ such that

$$
\left\|\left(\mathbf{V}_{\alpha, p} f\right)^{p-1}\right\|_{L^{B}(\Omega)} \leq C\|f\|_{L^{A}(\Omega)}
$$

for every $f \in L^{A}(\Omega)$.

In particular, if $|\Omega|<\infty$, then inequality (3.11) holds even if (3.10) is just fulfilled for $t \geq t_{0}$ for some $t_{0}>0$ and with $A$ replaced, if necessary, by an equivalent Young function near infinity making $E_{\alpha, p}$ finite. In this case, the constant $C$ in (3.11) depends also on A.

Example 3.3. Assume that $|\Omega|<\infty$ and that

$$
A(t) \approx t^{q}(\log t)^{\beta}
$$

near infinity, where either $q>1$ and $\beta \in \mathbb{R}$, or $q=1$ and $\beta \geq 0$. An application of Theorem 3.2 tells us that

$$
\left\|\left(\mathbf{V}_{\alpha, p} f\right)^{p-1}\right\|_{L^{B}(\Omega)} \leq C\|f\|_{L^{A}(\Omega)}
$$


for every $f \in L^{A}(\Omega)$, where

$$
B(t) \approx \begin{cases}t^{\frac{n}{n-\alpha p}}(\log t)^{\frac{n \beta}{n-\alpha p}-1} & \text { if } q=1 \text { and } \beta \geq 0, \\ t^{\frac{n q}{n-\alpha p q}}(\log t)^{\frac{n \beta}{n-\alpha p q}} & 1<q<\frac{n}{\alpha p} \text { and } \beta \in \mathbb{R}, \\ e^{\frac{n}{n(p-1)-\alpha(\beta+1) p}} & \text { if } q=\frac{n}{\alpha p}, \frac{n}{\alpha p^{\prime}}>1 \text { and } \beta<\frac{n}{\alpha p^{\prime}}-1, \\ e^{t^{\frac{n}{n(p-1)-\alpha p}}} & \text { if } q=\frac{n}{\alpha p}, \frac{n}{\alpha p^{\prime}}>1 \text { and } \beta=\frac{n}{\alpha p^{\prime}}-1, \\ e^{t^{-\frac{n}{\alpha \beta p}}} & \text { if } q=\frac{n}{\alpha p}, \frac{n}{\alpha p^{\prime}} \leq 1 \text { and } \beta<0,\end{cases}
$$

near infinity. In the remaining cases, namely if either $q>\frac{n}{\alpha p}$ and $\beta \in \mathbb{R}$, or $q=\frac{n}{\alpha p}, \frac{n}{\alpha p^{\prime}}>1$ and $\beta>\frac{n}{\alpha p^{\prime}}-1$, or $q=\frac{n}{\alpha p}, \frac{n}{\alpha p^{\prime}} \leq 1$ and $\beta \geq 0$, then

$$
\left\|\left(\mathbf{V}_{\alpha, p} f\right)^{p-1}\right\|_{L^{\infty}(\Omega)} \leq C\|f\|_{L^{A}(\Omega)}
$$

for every $f \in L^{A}(\Omega)$.

The proof of Theorem 3.2 relies upon a special case of the interpolation result contained in Theorem 3.4 below. In analogy with (3.8) and (3.9), its statement involves the functions $E, F:[0, \infty) \rightarrow[0, \infty]$ associated with $\sigma \in(1, \infty)$, $\varrho \in(0, \infty)$ and with the Young functions $A$ and $B$ as

$$
E(t)= \begin{cases}\left(\int_{0}^{t}\left(\frac{\tau^{\varrho-1+\frac{\varrho}{\sigma}}}{A(\tau)^{\frac{\varrho}{\sigma}}}\right)^{\frac{\sigma}{\sigma-\varrho}} d \tau\right)^{\frac{1}{\varrho}-\frac{1}{\sigma}} & \text { if } \varrho<\sigma, \\ \sup _{\tau \in(0, t)} \frac{\tau}{A(\tau)^{\frac{1}{\sigma}}} & \text { if } \varrho \geq \sigma,\end{cases}
$$

and

$$
F(t)=\left(\int_{0}^{t} \frac{B(\tau)}{\tau^{1+\sigma^{\prime}}} d \tau\right)^{\frac{1}{\sigma^{\prime}}}
$$

for $t \geq 0$.

Theorem 3.4. Let $1<\sigma<\infty, 0<\varrho<\infty$ and let $A$ and $B$ be Young functions. Let $\Omega$ be a measurable subset of $\mathbb{R}^{n}$. Assume that the function $E$ defined by (3.12) is finite-valued for $t>0$, and there exists a constant $\gamma>0$ such that

$$
F\left(\frac{E(t)}{\gamma}\right) \leq \gamma \frac{A(t)}{t} \quad \text { for } t>0 .
$$

Then $L^{A}(\Omega) \subset L^{1}(\Omega)+L^{\sigma, \varrho}(\Omega)$. Moreover, if $T$ is a quasi-linear operator such that

$$
T: L^{1}(\Omega) \rightarrow L^{\sigma^{\prime}, \infty}(\Omega)
$$


and

$$
T: L^{\sigma, \varrho}(\Omega) \rightarrow L^{\infty}(\Omega)
$$

then

$$
T: L^{A}(\Omega) \rightarrow L^{B}(\Omega)
$$

The norm of $T$ in (3.17) depends on the norms of $T$ in (3.15) and (3.16), on $\sigma, \varrho$, $\gamma$, and on the constant $c$ appearing in (2.13).

Remark 3.5. If $|\Omega|<\infty$, then, by (3.7), replacing $A$ with an equivalent Young function near infinity leaves $L^{A}(\Omega)$ unchanged (up to equivalent norms). Thus, the conclusions of Theorem 3.4 hold even if $A$ is replaced (if necessary) by some Young function equivalent to $A$ near infinity, which makes $E$ finite and inequality (3.14) true. In this case, however, the norm of $T$ in (3.17) depends also on $A$.

Proof of Theorem 3.2. By (2.31) and (2.32), the conclusions are a consequence of Theorem 3.4 and Remark 3.5 applied with $T=\left(\mathbf{V}_{\alpha, p}\right)^{p-1}, \sigma=\frac{n}{\alpha p}$ and $\varrho=\frac{1}{p-1}$.

Proof of Theorem 3.4. We begin by showing that, if $E(t)$ is finite for $t>0$, then $L^{A}(\Omega) \subset L^{1}(\Omega)+L^{\sigma, \varrho}(\Omega)$, and hence $T$ is well defined on $L^{A}(\Omega)$. To this purpose, it suffices to prove that if $f: \Omega \rightarrow \mathbb{R}$ is measurable and such that

$$
\int_{\Omega} A(|f(x)|) d x<\infty
$$

and the functions $f_{t}$ and $f^{t}$ are defined for $t>0$ as $f_{t}=\operatorname{sign}(f) \min \{t,|f|\}$ and $f^{t}=f-f_{t}$, then $f^{t} \in L^{1}(\Omega)$ and $f_{t} \in L^{\sigma, \varrho}(\Omega)$.

To verify these properties, note that if $a:[0, \infty) \rightarrow[0, \infty)$ is a nondecreasing function such that

$$
A(t)=\int_{0}^{t} a(\tau) d \tau \quad \text { for } t \geq 0,
$$

then

$$
\int_{\Omega} A(|f(x)|) d x=\int_{0}^{\infty} a(t)|\{|f|>t\}| d t .
$$

Thus,

$$
\begin{aligned}
\left\|f^{t}\right\|_{L^{1}(\Omega)} & =\int_{t}^{\infty}|\{|f|>\tau\}| d \tau=\int_{t}^{\infty} a(\tau)|\{|f|>\tau\}| \frac{d \tau}{a(\tau)} \\
& \leq \frac{1}{a(t)} \int_{t}^{\infty} a(\tau)|\{|f|>\tau\}| d \tau \\
& \leq \frac{1}{a(t)} \int_{\Omega} A(|f(x)|) d x \quad \text { for } t>0 .
\end{aligned}
$$

Hence, $f^{t} \in L^{1}(\Omega)$. Here we have exploited the fact that $a(t)>0$ for $t>0$, since $E(t)<\infty$ for $t>0$. 
Next, observe that

$$
\|g\|_{L^{\sigma, \varrho}(\Omega)}=\left(\sigma \int_{0}^{\infty}|\{|g|>\tau\}|^{\frac{\varrho}{\sigma}} \tau^{\varrho-1} d \tau\right)^{\frac{1}{\varrho}}
$$

for every $g \in L^{\sigma, \varrho}(\Omega)$. Therefore,

$$
\begin{aligned}
\left\|f_{t}\right\|_{L^{\sigma, \varrho}(\Omega)} & =\left(\sigma \int_{0}^{t}|\{|f|>\tau\}|^{\frac{\varrho}{\sigma}} \tau^{\varrho-1} d \tau\right)^{\frac{1}{\varrho}} \\
& \leq C E(t)\left(\int_{0}^{t} \frac{A(\tau)}{\tau}|\{|f|>\tau\}| d \tau\right)^{\frac{1}{\sigma}} \\
& \leq C E(t)\left(\int_{0}^{t} a(\tau)|\{|f|>\tau\}| d \tau\right)^{\frac{1}{\sigma}} \\
& \leq C E(t)\left(\int_{\Omega} A(|f(x)|) d x\right)^{\frac{1}{\sigma}} \quad \text { for } t>0
\end{aligned}
$$

for some constant $C=C(\sigma, \varrho)$. Note that the first inequality in (3.21) follows from a characterization of embeddings between generalized spaces of Lorentz type - see e.g. [9, Theorem 3.1], and the second one from the fact that $A(t) \leq t a(t)$ for $t \geq 0$. This shows that $f_{t} \in L^{\sigma, \varrho}(\Omega)$.

We now establish (3.17). Let $f$ be any function fulfilling (3.18).

Let $b:[0, \infty) \rightarrow[0, \infty)$ be a non-decreasing function such that

$$
B(t)=\int_{0}^{t} b(\tau) d \tau \quad \text { for } t \geq 0
$$

Define

$$
\zeta(t)=E^{-1}(\gamma t) \quad \text { for } t \geq 0,
$$

where $E^{-1}:[0, \infty) \rightarrow[0, \infty]$ denotes the generalized right-continuous inverse of $E$. Let $t_{0}=\sup \{t \geq 0: B(t)=0\}$. Owing to (3.12) and (3.14), one has that $E(0) \leq \gamma t_{0}$. Hence, if $E$ is continued to $[0, \infty]$ by $E(\infty)=\lim _{s \rightarrow \infty} E(s)$, then

$$
E\left(E^{-1}(\gamma t)\right) \leq \gamma t \quad \text { if } t_{0} \leq t
$$

and, in fact,

$$
E\left(E^{-1}(\gamma t)\right)=\gamma t \quad \text { if } t_{0} \leq t<E(\infty) / \gamma .
$$

Denote by $M_{1}$ and $M_{2}$ the norms of the operator $T$ in (3.15) and (3.16), respectively. Then,

$$
\left|\left\{\left|T f^{\zeta(t)}\right|>\vartheta\right\}\right| \leq\left(\frac{M_{1}}{\vartheta}\right)^{\sigma^{\prime}}\left(\int_{\zeta(t)}^{\infty}|\{|f|>\tau\}| d \tau\right)^{\sigma^{\prime}} \quad \text { for } t, \vartheta>0
$$


and

$$
\left\|T f_{\zeta(t)}\right\|_{L^{\infty}(\Omega)} \leq M_{2}\left(\sigma \int_{0}^{\zeta(t)}|\{|f|>\tau\}|^{\frac{\varrho}{\sigma}} \tau^{\varrho-1} d \tau\right)^{\frac{1}{\varrho}} \quad \text { for } t>0 .
$$

Given any positive constant $K$, one has that

$$
\begin{aligned}
\int_{\Omega} B\left(\frac{|T f(y)|}{4 c K}\right) d y= & \int_{0}^{\infty} b(t)|\{|T f|>4 c K t\}| d t \\
\leq & \int_{0}^{\infty} \frac{B(t)}{t}|\{|T f|>2 c K t\}| d t \\
\leq & \int_{0}^{\infty} \frac{B(t)}{t}\left|\left\{\left|T f_{\zeta(t)}\right|>K t\right\}\right| d t \\
& +\int_{0}^{\infty} \frac{B(t)}{t}\left|\left\{\left|T f^{\zeta(t)}\right|>K t\right\}\right| d t
\end{aligned}
$$

where the first inequality holds since $b(t) \leq B(2 t) / t$ for $t>0$. By (3.26), (3.21) (with $t$ replaced by $\zeta(t)),(3.22)$, and (3.23),

$$
\begin{aligned}
\left\|T f_{\zeta(t)}\right\|_{L^{\infty}(\Omega)} & \leq C M_{2} E(\zeta(t))\left(\int_{\Omega} A(|f(x)|) d x\right)^{\frac{1}{\sigma}} \\
& \leq C M_{2} \gamma t\left(\int_{\Omega} A(|f(x)|) d x\right)^{\frac{1}{\sigma}} \quad \text { for } t>t_{0},
\end{aligned}
$$

for some constant $C=C(\sigma, \varrho)$. On choosing

$$
K=\gamma C M_{2}\left(\int_{\Omega} A(|f(x)|) d x\right)^{\frac{1}{\sigma}}
$$

we thus have that

$$
\left|\left\{\left|T f_{\zeta(t)}\right|>K t\right\}\right|=0 \quad \text { for } t>t_{0} .
$$

Equation (3.30), combined with the fact that $B(t)=0$ if $0 \leq t \leq t_{0}$, entails that the first addend on the rightmost side of (3.27) vanishes.

As far as the second addend is concerned, from (3.25) we deduce that

$$
\begin{aligned}
& \int_{0}^{\infty} \frac{B(t)}{t}\left|\left\{\left|T f^{\zeta(t)}\right|>K t\right\}\right| d t \\
& \quad \leq\left(\frac{M_{1}}{K}\right)^{\sigma^{\prime}} \int_{0}^{\infty} \frac{B(t)}{t^{1+\sigma^{\prime}}}\left(\int_{\zeta(t)}^{\infty}|\{|f|>\tau\}| d \tau\right)^{\sigma^{\prime}} d t
\end{aligned}
$$


On the other hand, by (3.14), (3.22) and (3.24),

$$
\left(\int_{0}^{t} \frac{B(\tau)}{\tau^{1+\sigma^{\prime}}} d \tau\right)^{\frac{1}{\sigma^{\prime}}}\left\|\frac{\tau}{A(\tau)}\right\|_{L^{\infty}(\zeta(t), \infty)}=F(t) \frac{\zeta(t)}{A(\zeta(t))} \leq \gamma \quad \text { if } t<E(\infty) / \gamma
$$

Here, we have again exploited the fact that $B(t)=0$ if $0 \leq t \leq t_{0}$. Thus, since $\zeta(t)=\infty$ if $t \geq E(\infty) / \gamma$, by a variant of a classical weighted Hardy inequality (see e.g. [12, Lemma1]),

$$
\int_{0}^{\infty} \frac{B(t)}{t^{1+\sigma^{\prime}}}\left(\int_{\zeta(t)}^{\infty}|\{|f|>\tau\}| d \tau\right)^{\sigma^{\prime}} d t \leq C\left(\int_{0}^{\infty} \frac{A(\tau)}{\tau}|\{|f|>\tau\}| d \tau\right)^{\sigma^{\prime}}
$$

for some constant $C=C(\sigma, \gamma)$. Coupling (3.31) with (3.33) yields

$$
\begin{aligned}
\int_{0}^{\infty} \frac{B(t)}{t}\left|\left\{\left|T f^{\zeta(t)}\right|>K t\right\}\right| d t & \leq C\left(\frac{M_{1}}{K}\right)^{\sigma^{\prime}}\left(\int_{0}^{\infty} \frac{A(t)}{t}|\{|f|>t\}| d t\right)^{\sigma^{\prime}} \\
& \leq C\left(\frac{M_{1}}{K}\right)^{\sigma^{\prime}}\left(\int_{0}^{\infty} a(t)|\{|f|>t\}| d t\right)^{\sigma^{\prime}} \\
& =C\left(\frac{M_{1}}{K}\right)^{\sigma^{\prime}}\left(\int_{\Omega} A(|f(x)|) d x\right)^{\sigma^{\prime}} .
\end{aligned}
$$

Altogether, we infer that

$$
\int_{\Omega} B\left(\frac{|T f(y)|}{\gamma c M_{2} C\left(\int_{\Omega} A(|f(x)|) d x\right)^{\frac{1}{\sigma}}}\right) d y \leq C\left(\frac{M_{1}}{K}\right)^{\sigma^{\prime}}\left(\int_{\Omega} A(|f(x)|) d x\right)^{\sigma^{\prime}}
$$

for some constant $C=C(\sigma, \varrho)$. Hence, (3.17) easily follows.

\section{Estimates for local solutions to elliptic equations}

Results of the preceding sections are exploited here to derive estimates for local solutions to nonlinear elliptic equations having the form

$$
-\operatorname{div}(a(x, \nabla u))=f \quad \text { in } \Omega \text {. }
$$

Here, $\Omega$ is an open subset of $\mathbb{R}^{n}, f \in L^{1}(\Omega)$, and $a: \Omega \times \mathbb{R}^{n} \rightarrow \mathbb{R}^{n}$ is a Cararhéodory vector field such that

$$
\left\{\begin{array}{l}
|a(x, \xi)| \leq \Lambda\left(|\xi|^{p-1}+\lambda^{p-1}\right) \\
a(x, \xi) \cdot \xi \geq \Lambda^{-1}\left(|\xi|^{p}-\lambda^{p}\right)
\end{array}\right.
$$


for some $p>1$, for some constants $\lambda \geq 0$ and $\Lambda>0$, for $\xi \in \mathbb{R}^{n}$ and for a.e. $x \in \Omega$. Here, the dot "." stands for scalar product in $\mathbb{R}^{n}$. Clearly, the standard p-Laplace equation (1.5) falls within this framework.

A function $u \in W_{\text {loc }}^{1, p}(\Omega)$ is called a local weak solution to equation (4.1) if

$$
\int_{\Omega^{\prime}} a(x, \nabla u) \cdot \nabla \phi d x=\int_{\Omega^{\prime}} f \phi d x
$$

for every open set $\Omega^{\prime} \subset \subset \Omega$ and every $\phi \in W_{0}^{1, p}\left(\Omega^{\prime}\right)$.

Our rearrangement estimates rely upon bounds for solutions to (4.1) and for their gradient involving Wolff potentials of $f$. Given $p \in(1, \infty)$ and $\alpha>0$, the Wolff potential $\mathbf{W}_{\alpha, p} f$ of a measurable function $f$ in $\Omega$ (continued by 0 outside $\Omega$ ) is defined as

$$
\mathbf{W}_{\alpha, p} f(x)=\int_{0}^{\infty}\left(r^{-n+\alpha p} \int_{B(x, r)}|f(y)| d y\right)^{\frac{1}{p-1}} \frac{d r}{r} \quad \text { for } x \in \mathbb{R}^{n} .
$$

Here, $B(x, r)$ denotes the ball centered at $x$ and with radius $r$. The Wolff potential $\mathbf{W}_{\alpha, p} f$ admits an estimate from above, independent of $f$, in terms of the nonlinear potential $\mathbf{V}_{\alpha, p} f$. Indeed, by [3, Theorem 3.1], if $\alpha p<n$ there exists a constant $C=C(\alpha, p, n)$ such that

$$
\mathbf{W}_{\alpha, p} f(x) \leq C \mathbf{V}_{\alpha, p} f(x) \quad \text { for } x \in \mathbb{R}^{n} .
$$

Incidentally, let us mention that a reverse pointwise inequality only holds for $p, n$ and $\alpha$ in a suitable range - see [20]. However, a lower bound in integral form for $\mathbf{W}_{\alpha, p} f$ in terms of $\mathbf{V}_{\alpha, p} f$ is always available, as proved in [21].

Let us first focus on bounds for $u$. As a consequence of [24, Theorem 1.6] (see also [26, Theorem 4.36]), for every $q>p-1$ there exists a positive constant $C=C(p, q, n, \Lambda)$ such that if $f \in W^{-1, p^{\prime}}(\Omega)$, and $u \in W_{\text {loc }}^{1, p}(\Omega)$ is a local weak solution to equation (4.1), then

$$
|u(x)| \leq C\left(\frac{1}{|B(x, R)|} \int_{B(x, R)}(|u|+\lambda)^{q} d y\right)^{\frac{1}{q}}+C \mathbf{W}_{1, p} f(x)
$$

for a.e. $x \in \Omega$ such that $B(x, 2 R) \subset \Omega$.

Combining estimates (4.5) and (4.4) with Theorem 2.1 yields the following local bound for the decreasing rearrangement of a solution $u$ to (4.1) in terms of the decreasing rearrangement of $f$ and of the $L^{q}$-norm of $u$.

Theorem 4.1. Let $1<p<n$. Assume that (4.2) holds. Let $f \in W^{-1, p^{\prime}}(\Omega)$ and let $u \in W_{\mathrm{loc}}^{1, p}(\Omega)$ be a local weak solution to equation (4.1). Then for any bounded open subsets $\Omega^{\prime \prime} \subset \subset \Omega^{\prime} \subset \subset \Omega$ there exist constants $C=C\left(p, q, n, \Lambda, \Omega^{\prime}, \Omega^{\prime \prime}\right)$ and $k=k(p, n)$ such that

$$
\left(u_{\mid \Omega^{\prime \prime}}\right)^{*}(s) \leq C \lambda+C\|u\|_{L^{q}\left(\Omega^{\prime}\right)}+C \int_{k s}^{\infty} r^{-1+\frac{p^{\prime}}{n}} f^{* *}(r)^{\frac{1}{p-1}} d r \quad \text { for } s>0 .
$$


Owing to Theorem 4.1, estimates for rearrangement invariant quasi-norms of solutions to equation (4.1) are reduced to one-dimensional Hardy type inequalities.

Corollary 4.2. Let $p, q, f, u, \Omega, \Omega^{\prime}$ and $\Omega^{\prime \prime}$ be as in Theorem 4.1. Let $X(\Omega)$ and $Y(\Omega)$ be rearrangement invariant quasi-normed spaces. Assume that either (2.7) or (2.8)-(2.9) hold with $\alpha=1$. Then there exists a constant $C$ such that

$$
\left\||u|^{p-1}\right\|_{Y\left(\Omega^{\prime \prime}\right)} \leq C+C\|u\|_{L^{q}\left(\Omega^{\prime}\right)}^{p-1}+C\|f\|_{X(\Omega)}
$$

for every $f \in X(\Omega)$.

In the special case when $\lambda=0$ in (4.9) and $\Omega=\mathbb{R}^{n}$, one can let $R \rightarrow \infty$ in (4.5). Estimate (4.6) then takes a simplified form. In particular, the next result holds for solutions to the $p$-Laplace equation in $\mathbb{R}^{n}$.

Theorem 4.3. Let $1<p<n$. Let $f \in W^{-1, p^{\prime}}\left(\mathbb{R}^{n}\right)$ and let $u$ be a local weak solution to (1.5) such that $u \in L^{q}\left(\mathbb{R}^{n}\right)$ for some $q \in(p-1, \infty)$. Then there exist constants $C=C(p, n)$ and $k=k(p, n)$ such that

$$
u^{*}(s) \leq C \int_{k s}^{\infty} r^{-1+\frac{p^{\prime}}{n}} f^{* *}(r)^{\frac{1}{p-1}} d r \quad \text { for } s>0 .
$$

We are now concerned with gradient estimates. They require stronger structure assumptions on the differential operator in (4.1). In the spirit of [18], we assume that $a(x, \cdot) \in C^{1}\left(\mathbb{R}^{n}\right)$ for $x \in \Omega$, and that its gradient $a_{\xi}$ in the variable $\xi \in \mathbb{R}^{n}$ is a Cararhéodory function. Moreover, we require that there exist constants $\lambda \geq 0$ and $\Lambda>0$ such that

$$
\left\{\begin{array}{l}
|a(x, \xi)|+\left|a_{\xi}(x, \xi)\right|\left(|\xi|^{2}+\lambda^{2}\right)^{\frac{1}{2}} \leq \Lambda\left(|\xi|^{2}+\lambda^{2}\right)^{\frac{p-1}{2}} \\
a_{\xi}(x, \xi) \eta \cdot \eta \geq \Lambda^{-1}\left(|\xi|^{2}+\lambda^{2}\right)^{\frac{p-2}{2}} \\
|a(x, \xi)-a(y, \xi)| \leq \Lambda \omega(|x-y|)\left(|\xi|^{2}+\lambda^{2}\right)^{\frac{p-1}{2}}
\end{array}\right.
$$

for $x, y \in \Omega$ and $\xi, \eta \in \mathbb{R}^{n}$. Here, $\omega:[0, \infty) \rightarrow[0,1]$ is a non-decreasing concave function such that

$$
\int_{0} \frac{\omega(r)^{\frac{2}{p}}}{r} d r<\infty
$$

Theorem 4.4 below provides us with a rearrangement estimate for the gradient of local solutions to (4.1), when $p \geq 2$. This result follows via a key bound contained in [18, Theorem 5.2], ensuring that if $p \geq 2$, then there exist positive constants $C=C(p, n, \Lambda)$ and $R_{0}=R_{0}(p, n, \Lambda, \omega(\cdot))$ such that if $f \in W^{-1, p^{\prime}}(\Omega)$ and $u \in W_{\text {loc }}^{1, p}(\Omega)$ is a local weak solution to equation (4.1), then

$$
|\nabla u(x)| \leq \frac{C}{|B(x, R)|} \int_{B(x, R)}(|\nabla u|+\lambda) d y+C \mathbf{W}_{\frac{1}{p}, p} f(x)
$$

for $R<R_{0}$ and for a.e. $x \in \Omega$ such that $B(x, 2 R) \subset \Omega$. Moreover, if $a(x, \xi)$ is independent of $x$, then the same conclusion is true with $R_{0}=\infty$. 
Theorem 4.4. Let $p \geq 2$. Assume that (4.9) holds. Let $f \in W^{-1, p^{\prime}}(\Omega)$ and let $u \in W_{\text {loc }}^{1, p}(\Omega)$ be a local weak solution to equation (4.1). Then for any bounded open subsets $\Omega^{\prime \prime} \subset \subset \Omega^{\prime} \subset \subset \Omega$ there exist constants $C=C\left(p, n, \Lambda, \omega, \Omega^{\prime}, \Omega^{\prime \prime}\right)$ and $k=k(p, n)$ such that

$$
\begin{aligned}
\left(|\nabla u|_{\mid \Omega^{\prime \prime}}\right)^{*}(s) \leq & C \lambda+C\|\nabla u\|_{L^{1}\left(\Omega^{\prime}\right)} \\
& +C \int_{k s}^{\infty} r^{-1+\frac{1}{n(p-1)}} f^{* *}(r)^{\frac{1}{p-1}} d r \quad \text { for } s>0 .
\end{aligned}
$$

Bounds for general rearrangement invariant quasi-norms of $|\nabla u|$ can be derived from Theorem 4.4.

Corollary 4.5. Let $p, f, u, \Omega, \Omega^{\prime}$ and $\Omega^{\prime \prime}$ be as in Theorem 4.4. Let $X(\Omega)$ and $Y(\Omega)$ be rearrangement invariant quasi-normed spaces. Assume that either (2.7) or (2.8)-(2.9) hold with $\alpha=\frac{1}{p}$. Then there exists a constant $C$ such that

$$
\left\||\nabla u|^{p-1}\right\|_{Y\left(\Omega^{\prime \prime}\right)} \leq C+C\|\nabla u\|_{L^{1}\left(\Omega^{\prime}\right)}^{p-1}+C\|f\|_{X(\Omega)}
$$

for every $f \in X(\Omega)$.

The counterpart of Theorem 4.3 for $|\nabla u|$ is the content of the following result.

Theorem 4.6. Let $p \geq 2$. Let $f \in W^{-1, p^{\prime}}\left(\mathbb{R}^{n}\right)$ and let $u$ be a local solution to (1.5) such that $|\nabla u| \in L^{q}\left(\mathbb{R}^{n}\right)$ for some $q \in[1, \infty)$. Then there exist constants $C=C(p, n)$ and $k=k(p, n)$ such that

$$
|\nabla u|^{*}(s) \leq C \int_{k s}^{\infty} r^{-1+\frac{1}{n(p-1)}} f^{* *}(r)^{\frac{1}{p-1}} d r \quad \text { for } s>0 .
$$

Bounds in Lorentz and Orlicz spaces for local solutions $u$ to equation (4.1) and for their gradient follow via Theorem 4.1 and Corollary 4.2, and Theorem 4.4 and Corollary 4.5, respectively, in the same way as Theorems 3.1 and 3.2 follow from Theorem 2.1 and Corollary 2.3.

The relevant bounds for $u$ take the following form.

Theorem 4.7. Let $p$ and $u$ be as in Theorem 4.1. Assume that

$$
f \in L_{\mathrm{loc}}^{\sigma, \varrho}(\Omega) \text {. }
$$

(i) If $0<\varrho \leq \infty$ and $1<\sigma<\frac{n}{p}$, then

$$
u \in L_{\mathrm{loc}}^{\frac{\sigma n(p-1)}{n-\sigma p}, \varrho(p-1)}
$$

(ii) If $\sigma=1$ and $0<\varrho \leq 1$, then

$$
u \in L_{\mathrm{loc}}^{\frac{n(p-1)}{n-p}, \infty}(\Omega) .
$$


(iii) If $\sigma=\frac{n}{p}$ and $\varrho>\frac{1}{p-1}$, then

$$
u \in L^{\infty, \varrho(p-1)}(\log L)_{\operatorname{loc}}^{-1}(\Omega) .
$$

(iv) If $\sigma=\frac{n}{p}$ and $\varrho \leq \frac{1}{p-1}$, then

$$
u \in L_{\mathrm{loc}}^{\infty}(\Omega) .
$$

Special cases of Theorem 4.7 for solutions to linear homogeneous Dirichlet problems go back to [38]. As far as nonlinear Dirichlet problems are concerned, estimates for solutions in Lorentz spaces can be found in [8] and [4]. In particular, in the latter paper Neumann problems are considered as well, and the sharpness of the relevant estimates is established. Local estimates corresponding to Case (i) of Theorem 4.7 are contained in [31, Theorem 15].

Theorem 4.8. Let $p$ and $u$ be as in Theorem 4.1. Let $A$ and $B$ be Young functions. Assume that $A$ is modified near 0 , if necessary, in such a way that the function

$$
E_{1, p}(t)= \begin{cases}\left(\int_{0}^{t} \frac{\tau^{\frac{n}{n(p-1)-p}-1}}{A(\tau)^{\frac{p}{n(p-1)-p}}} d \tau\right)^{\frac{n(p-1)-p}{n}} & \text { if } n>p^{\prime}, \\ \sup _{\tau \in(0, t)} \frac{\tau}{A(\tau)^{\frac{p}{n}}} & \text { if } n \leq p^{\prime}\end{cases}
$$

is finite-valued for $t \geq 0$. Let

$$
F_{1, p}(t)=\left(\int_{0}^{t} \frac{B(\tau)}{\tau^{1+\frac{n}{n-p}}} d \tau\right)^{\frac{n-p}{n}} \quad \text { for } t \geq 0 .
$$

Assume that there exist $\gamma>0$ and $t_{0}>0$ such that

$$
F_{1, p}\left(\frac{E_{1, p}(t)}{\gamma}\right) \leq \gamma \frac{A(t)}{t} \quad \text { for } t>t_{0} .
$$

If $f \in L_{\mathrm{loc}}^{A}(\Omega)$, then $|u|^{p-1} \in L_{\mathrm{loc}}^{B}(\Omega)$.

Estimates in Orlicz spaces for solutions to homogeneous Dirichlet problems for linear elliptic equations, in the spirit of Theorem 4.8, can be found in [13].

We conclude with gradient bounds in Lorentz and Orlicz spaces.

Theorem 4.9. Let $p$ and $u$ be as in Theorem 4.4. Assume that

$$
f \in L_{\mathrm{loc}}^{\sigma, \varrho}(\Omega) .
$$


(i) If $0<\varrho \leq \infty$ and $1<\sigma<n$, then

$$
|\nabla u| \in L_{\mathrm{loc}}^{\frac{\sigma n(p-1)}{n-\sigma}, \varrho(p-1)}(\Omega) .
$$

(ii) If $\sigma=1$ and $0<\varrho \leq 1$, then

$$
|\nabla u| \in L_{\mathrm{loc}}^{\frac{n(p-1)}{n-1}, \infty}(\Omega) .
$$

(iii) If $\sigma=n$ and $\varrho>\frac{1}{p-1}$, then

$$
|\nabla u| \in L^{\infty, \varrho(p-1)}(\log L)_{\operatorname{loc}}^{-1}(\Omega) .
$$

(iv) If $\sigma=n$ and $\varrho \leq \frac{1}{p-1}$, then

$$
|\nabla u| \in L_{\mathrm{loc}}^{\infty}(\Omega) .
$$

Some instances of Cases (i) and (ii) of Theorem 4.9 are contained in [18,31]. Gradient bounds in Marcinkiewicz spaces, namely Lorentz spaces whose second index is infinity, and in Morrey spaces, are also established in [30], where a nonlinear Calderón-Zygmund theory involving fractional-order Sobolev spaces in developed.

Theorem 4.10. Let $p$ and $u$ be as in Theorem 4.4 Let A and B be Young functions. Assume that $A$ is modified near 0 , if necessary, in such a way that the function

$$
E_{\frac{1}{p}, p}(t)=\left(\int_{0}^{t} \frac{\tau^{\frac{n}{n(p-1)-1}-1}}{A(\tau)^{\frac{1}{n(p-1)-1}}} d \tau\right)^{\frac{n(p-1)-1}{n}}
$$

is finite-valued for $t \geq 0$. Let

$$
F_{\frac{1}{p}, p}(t)=\left(\int_{0}^{t} \frac{B(\tau)}{\tau^{1+n^{\prime}}} d \tau\right)^{\frac{1}{n^{\prime}}} \quad \text { for } t \geq 0
$$

Assume that there exist $\gamma>0$ and $t_{0}>0$ such that

$$
F_{\frac{1}{p}, p}\left(\frac{E_{\frac{1}{p}, p}(t)}{\gamma}\right) \leq \gamma \frac{A(t)}{t} \quad \text { for } t>t_{0}
$$

If $f \in L_{\mathrm{loc}}^{A}(\Omega)$, then $|\nabla u|^{p-1} \in L_{\mathrm{loc}}^{B}(\Omega)$.

From case (iv) of Theorem 4.9 we have, in particular, a Lorentz space condition on $f$ ensuring the local Lipschitz continuity of $u$. For solutions to the Laplace equation in $\mathbb{R}^{n}$, such a condition was found and shown to be sharp in the framework of rearrangement invariant spaces in [11].

Corollary 4.11. Let $p$ and $u$ be as in Theorem 4.4. If $f \in L_{\mathrm{loc}}^{n, \frac{1}{p-1}}(\Omega)$, then $u$ is locally Lipschitz continuous in $\Omega$. 


\section{References}

[1] D. R.Adams and L. I. Hedberg, "Function Spaces and Potential Theory", Springer, Berlin, 1996.

[2] D. R. ADAMS, Traces of potentials II, Indiana Univ. Math. J. 22 (1973), 907-918.

[3] D. R. AdAms and N. G. MEYERS, Thinnes and Wiener criteria for non-linear potentials, Indiana Univ. Math. J. 22 (1972), 132-158.

[4] A. Alberico and A. Cianchi, Optimal summability of solutions to nonlinear elliptic problems, Nonlinear Anal. 67 (2007), 1775-1790.

[5] A. Alvino, V. Ferone and G. Trombetti, Estimates for the gradient of solutions of nonlinear elliptic equations with $L^{1}$ data, Ann. Mat. Pura. Appl. 178 (2000), 129-142.

[6] A. Alvino, A. Cianchi, V. G. MaZ'Ya and A. Mercaldo, Well-posed elliptic Neumann problems involving irregular data and domains, Ann. Inst. H. Poincaré Anal. Non Linéaire 27 (2010), 1017-1054.

[7] C. Bennett and R. Sharpley, "Interpolation of Operators", Academic Press, Boston, 1988.

[8] M. F. BETTA, V. FERONE and A. MERCALDO, Regularity for solutions of nonlinear elliptic equations, Bull. Sci. Math. 118 (1994), 539-567.

[9] M. Carro, L. Pick, J. Soria and V. Stepanov, On embeddings between classical Lorentz spaces, Math. Inequal. Appl. 4 (2001), 397-428.

[10] M. CARRo and J. Soria, Boundedness of some integral operators, Canad. J. Math. 45 (1993), 1155-1166.

[11] A. CIANCHI, Maximizing the $L^{\infty}$ norm of the gradient of solutions to the Poisson equation, J. Geom. Anal. 2 (1992), 499-515.

[12] A. Cianchi, An optimal interpolation theorem of Marcinkiewicz type in Orlicz spaces, J. Funct. Anal. 153 (1998), 357-381.

[13] A. CiAnchI, Strong and weak type inequalities for some classical operators in Orlicz spaces, J. London Math. Soc. 60 (1999), 187-202.

[14] A. CianchI, Symmetrization and second order Sobolev inequalities, Ann. Mat. Pura Appl. 183 (2004), 45-77.

[15] A. CIANCHI, Sharp estimates for nonlinear potentials and applications, In: "Analyis, Partial Differential Equations, and Applications", A. Cialdea, F. Lanzara, P. E. Ricci (eds.), Birkhauser, Basel, 2009, 57-64.

[16] A. CIANCHI, R. KERMAN and L. PICK, Boundary trace inequalities and rearrangements, J. Anal. Math. 105 (2008), 241-265.

[17] F. DuZaAr and G. Mingione, Gradient estimates in non-linear potential theory, Atti Accad. Naz. Lincei Cl. Sci. Fis. Mat. Natur. Rend. Lincei (9) Mat. Appl. 20 (2009), 179190.

[18] F. DuZaAr and G. Mingione, Gradient estimates via non-linear potentials, Amer. J. Math., 39 (2010), 379-418.

[19] F. DuZAAR and G. Mingione, Gradient contunuity estimates, Cal. Var. Partial Differential Equations 13 (2011), 459-486.

[20] V. P. HaVin and V. G. MAZ'YA, Nonlinear potential theory, Usp. Mat. Nauk 27 (1972), 67-138 (Russian); English translation: Russian Math. Surveys 27 (1972), 71-148.

[21] L. I. Hedberg and Th. H. WolfF, Thin sets in nonlinear potential theory, Ann. Inst. Fourier (Grenoble) 33 (1983), 161-187.

[22] T. HolmstedT, Interpolation of quasi-normed spaces, Math. Scand. 26 (1970), 177-199.

[23] R. Kerman and L. PICK, Optimal Sobolev imbeddings, Forum Math. 18 (2006), 535-570.

[24] T. KILPELAINEN and J. MALÝ, The Wiener test and potential estimates for quasilinear elliptic equations, Acta Math. 172 (1994), 137-161.

[25] D. Labutin, Potential estimates for a class of fully nonlinear equations, Duke Math. J. 111 (2002), 1-49. 
[26] J. Malý and W. P. ZIEMER, "Fine Regularity of Solutions to Elliptic Partial Differential Equations", American Mathematical Society, Providence, 1997.

[27] V. G. MAZ'YA, Some estimates of solutions of second-order elliptic equations, Dokl. Akad. Nauk. SSSR 137 (1961), 1057-1059 (Russian); English translation: Soviet Math. Dokl. 2 (1961), 413-415.

[28] V. G. MAZ'YA, On weak solutions of the Dirichlet and Neumann problems, Trusdy Moskov. Mat. Obšč. 20 (1969), 137-172 (Russian); English translation: Trans. Moscow Math. Soc. 20 (1969), 135-172.

[29] V. G. MAZ'YA, “Sobolev Spaces”, Springer-Verlag, Berlin, 1985.

[30] G. Mingione, The Calderon-Zygmund theory for elliptic problems with measure data, Ann. Sc. Norm. Super. Pisa, Cl. Sci. 6 (2007), 195-261.

[31] G. Mingione, Gradient estimates below the duality exponent, Math. Ann. 346 (2010), $571-627$.

[32] G. Mingione, Gradient potential estimates, J. Eur. Math. Soc. 13 (2011), 459-486.

[33] R. O'NeIL, Convolution operators in L ( $p, q)$ spaces, Duke Math. J. 30 (1963), 129-142.

[34] B. OPIC and L. PICK, On generalized Lorentz-Zygmund spaces, Math. Inequal. Appl. 2 (1999), 391-467.

[35] N. C. Phuc and I. E. Verbitsky, Quasilinear and Hessian equations of Lane-Emden type, Ann. of Math. 168 (2008), 859-914.

[36] N. C. PhUC and I. E. VerbitSKy, Singular quasilinear and Hessian equations and inequalities, J. Funct. Anal. 256 (2009), 1875-1906.

[37] R. S. STRICHARTZ, A note on Trudinger' s extension of Sobolev' s inequality, Indiana Univ. Math. J. 21 (1972), 841-842.

[38] G. Talenti, Elliptic equations and rearrangements, Ann. Sc. Norm. Super. Pisa (4) 3 (1976), 697-718.

[39] G. TALENTI, Nonlinear elliptic equations, rearrangements of functions and Orlicz spaces, Ann. Mat. Pura Appl. 120 (1979), 159-184.

[40] N. S. TRUdinger and X. J. WANG, On the weak continuity of elliptic operators and applications to potential theory, Amer. J. Math. 124 (2002), 369-410.

Dipartimento di Matematica "U. Dini" Università di Firenze

Piazza Ghiberti, 27

50122 Firenze, Italia

cianchi@unifi.it 